\title{
The effect of L-arginine supplementation on lipid profile: a systematic review and meta-analysis of randomised controlled trials
}

\author{
Amir Hadi ${ }^{1}$, Arman Arab², Sajjad Moradi ${ }^{3}$, Ana Pantovic ${ }^{4}$, Cain C. T. Clark ${ }^{5}$ and Ehsan Ghaedi ${ }^{6,7 *}$ \\ ${ }^{1}$ Halal Research Center of IRI, FDA, Tehran, Iran \\ ${ }^{2}$ Department of Community Nutrition, School of Nutrition and Food Science, Food Security Research Center, Isfahan University \\ of Medical Sciences, Isfahan 81745, Iran \\ ${ }^{3}$ Nutritional Sciences Department, School of Nutritional Sciences and Food Technology, Kermanshah University of Medical \\ Sciences, Kermanshah 6719851552, Iran \\ ${ }^{4}$ Institute for Medical Research, Centre of Research Excellence in Nutrition and Metabolism, University of Belgrade, \\ Belgrade 11000, Serbia \\ ${ }^{5}$ Faculty Research Centre for Sport, Exercise and Life Sciences, Coventry University, Coventry CV1 5FB, UK \\ ${ }^{6}$ Department of Cellular and Molecular Nutrition, School of Nutritional Sciences and Dietetics, Tehran University of Medical \\ Sciences, Tehran 14155-6446, Iran \\ ${ }^{7}$ Students' Scientific Research Center (SSRC), Tehran University of Medical Sciences (TUMS), Tehran 14155-6446, Iran
}

(Submitted 14 May 2019 - Final revision received 12 July 2019 - Accepted 15 July 2019)

\section{Abstract}

A number of clinical trials have examined the effect of L-arginine on lipid profile in recent years; however, the results remain equivocal. Therefore, the present study aims to summarise and quantitatively examine the available evidence on the effectiveness $\mathrm{L}$-arginine supplementation on lipid parameters using a systematic review and meta-analytic approach. Online databases including PubMed, Scopus, ISI Web of Science, Cochrane Library and Google Scholar were searched up to April 2019 for randomised controlled trials that examined the effect of L-arginine supplementation on lipid profile in adults. Treatment effects were expressed as weighted mean difference (WMD) and the corresponding standard error in concentrations of serum lipids. To estimate the overall effect of L-arginine supplementation, we used the random-effects model. In total, twelve studies were included in the systematic review. The meta-analysis revealed that $\mathrm{L}$-arginine supplementation did not significantly change the concentrations of total cholesterol (WMD: $-5.03 \mathrm{mg} / \mathrm{dl} ; 95 \% \mathrm{CI}-10 \cdot 78,0 \cdot 73 ; P=0 \cdot 08$; inconsistency index $\left(I^{2}\right)=39.0 \%$ ), LDL (WMD: $-0.47 \mathrm{mg} / \mathrm{dl} ; 95 \% \mathrm{CI}-3 \cdot 61,2 \cdot 66 ; P=0.76 ; I^{2}=0.0 \%$ ), or HDL (WMD: $0.57 \mathrm{mg} / \mathrm{dl} ; 95 \% \mathrm{CI}-1.28,2 \cdot 43 ; P=0.54 ;$ $I^{2}=68.4 \%$ ). A significant reduction was observed only in serum TAG levels (WMD: $-7.04 \mathrm{mg} / \mathrm{dl} ; 95 \%$ CI $-11.42,-2.67 ; P<0.001$; $I^{2}=0.0 \%$ ). This meta-analysis concludes that L-arginine supplementation can significantly reduce blood TAG levels; however, there is insufficient evidence to support its hypocholesterolaemic effects. To draw straightforward conclusions regarding generalised recommendations for L-arginine supplementation for improving lipid profile, there is a need for more well-controlled trials targeting exclusively patients with dyslipidaemia.

Key words: L-Arginine: Supplementation: Lipid profile: Systematic reviews: Meta-analyses

CVD are the leading causes of death among non-communicable diseases, posing a significant health and economic burden worldwide ${ }^{(1,2)}$. The American Heart Association reported that $17 \cdot 7$ million people (representing $31 \%$ of all global deaths) died from CVD in 2015, and this number is projected to rise to 23.6 million by $2030^{(3)}$. Dyslipidaemia has been identified as a major risk factor for $\mathrm{CVD}^{(4-6)}$. Thus, regulating and maintaining an optimal lipid profile is critical for the prevention of CVD. In this regard, statin therapy and diet modification are two of the most commonly prescribed approaches ${ }^{(7,8)}$. However, statins, among other commonly used lipid-lowering pharmacotherapies ${ }^{(9)}$, have been established to pose some serious adverse effects, such as myopathies and hepatotoxicity ${ }^{(10,11)}$. Thus, there is a demand to identify viable, anti-lipid agents that are able to pose cardioprotective effects without inducing any side effects.

L-Arginine is a semi-essential amino acid which our body derives either from dietary sources or from endogenous metabolism $^{(12,13)}$. L-Arginine is involved in several biochemical

Abbreviations: HDEL, hypoenergetic diet enriched in legumes; $I^{2}$, inconsistency index; RCT, randomised controlled trials; TC, total cholesterol; WMD, weighted mean difference.

* Corresponding author: E. Ghaedi, email Ehsanghaedi073@gmail.com 
Table 1. PICOS criteria used to perform the systematic review and meta-analysis

\begin{tabular}{ll}
\hline Parameter & Criteria \\
\hline Population & Adults \\
Intervention & Arginine \\
Comparator & Matched control group \\
Outcome & Circulating TC, TAG, LDL-cholesterol, and \\
Setting or study design & HDL-cholesterol \\
\hline
\end{tabular}

PICOS, participants, interventions, comparisons, outcomes and study design; TC, total cholesterol.

processes, including polyamine synthesis, ammonia detoxification, immune modulation and secretion of hormones such as glucagon and growth hormone and insulin ${ }^{(14-16)}$. What is more, this amino acid produces nitric oxide (NO), a key molecule involved in the regulation of cell metabolism, insulin signalling and secretion, neurotransmission and immune system function $^{(17,18)}$. It is suggested that L-arginine can be useful in improving lipid profile, due to its potential to increase NO production. Therefore, L-arginine has been investigated as a potentially cardio-protective compound, and seven meta-analyses concluded that it can be an effective tool in blood pressure management ${ }^{(19)}$. Several trials investigated the potential of L-arginine supplementation for the treatment of abnormal lipid profile; however, the results are inconsistent. For instance, some trials report that L-arginine supplementation induced a reduction in circulating concentrations of lipid parameters ${ }^{(20-23)}$, while others report no significant effect ${ }^{(18,24-30)}$. Discrepancies in the findings may conceivably be attributed to the differences in study designs, characteristics of study participants, duration and the supplementation dosage applied in the trials. Therefore, we conducted a meta-analysis of those randomised controlled trials (RCT) to examine the efficacy of L-arginine supplementation as a lipid-lowering agent.

\section{Methods}

We conducted and reported the present systematic review and meta-analysis following the preferred reporting items for systematic reviews and meta-analyses (PRISMA) statement (Supplementary Table S1) ${ }^{(31)}$ for identification, screening, eligibility and inclusion of articles. The present study was not prospectively registered. Participants, interventions, comparisons, outcomes and study design are shown in Table 1.

\section{Search strategy}

Two independent investigators (A. H. and E. G.) performed a systematic search of all articles published until April 2019 in the following online databases: PubMed, Scopus, Cochrane Library, ISI Web of Science and Google Scholar. We used the following Medical Subject Headings and corresponding keywords: ('arginine' OR 'L-arginine') AND ('lipid' OR 'hyperlipidaemia' OR 'dyslipidemia' OR 'cholesterol' OR 'CHOL' OR 'hypercholesterolaemia' OR 'lipoprotein' OR 'hyperlipoproteinemia' OR 'high density lipoprotein' OR 'HDL' OR 'low density lipoprotein' OR 'LDL' OR 'triglyceride' OR 'TG') AND ('Intervention Studies'
OR 'intervention' OR 'controlled trial' OR 'randomized' OR 'randomised' OR 'random' OR 'randomly' OR 'placebo' OR 'assignment'). The two reviewers also performed screening of the reference lists of relevant review articles and original papers that were selected for full-text review to identify potential eligible studies. Additionally, an email alert service was used to avoid missing any relevant articles. The language of the retrieved papers was restricted to English, while there were no restrictions regarding the year of publication.

\section{Study selection}

All studies retrieved from the electronic databases and reference lists were entered into endnote software (EndNote X6; Thomson Corporation) and duplicate studies were removed. In the next step, the titles and abstracts of the papers were examined by two independent reviewers (A. A. and E. G.) to exclude irrelevant articles. Afterwards, the full texts of the remaining publications were read and assessed according to the following four items: study design, participants, interventions and outcome measures. Finally, the studies were retained if they met the following inclusion criteria: (1) a randomised controlled design, (2) reporting the effect of L-arginine on at least one of the lipid profile parameters including total cholesterol (TC), TAG, LDLcholesterol, and HDL-cholesterol and (3) intervention for more than 4 weeks. Studies were excluded if they (1) involved L-arginine supplementation in combination with some drugs or other types of supplements (minerals, vitamins or herbal supplements, unless a separate arm controlled the effect of the mixed substance), (2) reported duplicate data (in this case, the data with complete follow-up and outcome measures were included), (3) included adolescents as the population and (4) were not peer-reviewed articles (protocol or conference proceeding). Any disagreements regarding the process of study selection were resolved in consultation with the principal investigator (A. H.).

\section{Data extraction}

The major demographic and clinical data from each of the selected studies were screened and extracted independently by two investigators (A. H. and E. G.) using a predesigned Excel sheet. Any controversy was solved via discussion with a third, independent researcher to reach a consensus. The extracted information was as follows: the first author's last name, publication year, study design, country, sample size, participants mean age, sex, baseline BMI, follow-up duration, intervention duration (in weeks), type of intervention, dose of L-arginine $(\mathrm{g} / \mathrm{d})$, type of control, health status of the participants and main results. Corresponding authors were contacted in case of any missing data.

\section{Quality assessment}

The Cochrane Collaboration tool ${ }^{(32)}$ was used for quality assessment, and it includes seven items, namely, randomisation sequence generation, allocation concealment, blinding of participants and personnel, blinding of outcome assessors, incomplete outcome data, selective reporting and other biases. Each domain 
was classified into three categories: low risk of bias, high risk of bias and unclear risk of bias. Finally, the overall quality of the studies was categorised into weak, fair or good, if $<3,3$ or $\geq 4$ domains were rated as low risk, respectively. Quality assessment was performed independently by two reviewers (A. A. and E. G.), while any disagreements were resolved by consulting the third reviewer (A. H.).

\section{Statistical analysis}

For carrying out the meta-analysis, we used STATA software (version 11; StataCorp). If outcome measures were reported in $\mathrm{mmol} / \mathrm{l}$, they were converted to $\mathrm{mg} / \mathrm{dl}$. The reviewers then extracted the mean difference between the baseline and endpoint data and the corresponding standard deviations in both intervention and control groups. If such data were not available, the mean difference was obtained by subtracting the mean value of the baseline point from that of the endpoint. If SD of the mean difference was not reported, it was calculated using the following formula: $\mathrm{SD}=$ square root $\left((\mathrm{SD} \text { pretreatment })^{2}+(\mathrm{SD}\right.$ post-treatment $)^{2}-(2 \times R \times \mathrm{SD}$ pretreatment $\times \mathrm{SD}$ post-treatment $\left.)\right)$. To ensure meta-analysis was not sensitive to the selected correlation coefficient $(R 0.5)$, all analyses were repeated using correlation coefficients of 0.2 and 0.8 . Where only a standard error was reported, SD was estimated using the following formula: $\mathrm{SD}=\mathrm{SE} \times \sqrt{ } n$ ( $n$ being the number of subjects in each group). Using random-effects model developed by DerSimonian \& Laird $^{(33)}$, the summary estimate was pooled as weighted mean difference (WMD) and 95\% CI. The inconsistency index $\left(I^{2}\right)$ was used to quantify statistical heterogeneity in the metaanalyses, and values greater than $50 \%$ were considered indicative of high heterogeneity. To identify the source of heterogeneity, subgroup analysis was conducted focusing on mean age, baseline BMI, dose of L-arginine supplementation, study duration and participant's health status. Sensitivity analysis was also performed to explore the extent to which inferences might depend on a particular study using the leave-one-out method (i.e. deleting one trail at a time and re-calculating the effect size). To assess publication bias, Begg( ${ }^{(34)}$ and Egger's ${ }^{(35)}$ regression tests were performed. In all statistical analyses, the level of significance was set at $P<0.05$.

\section{Ethical considerations}

Ethical issues (including plagiarism, misconduct, data fabrication, falsification, double publication or submission, redundancy) have been completely observed by the authors. There was no human or animal involvement in the present study.

\section{Results}

\section{Flow of study selection}

A total of 2216 publications were identified after the search of the electronic databases, out of which 755 were removed as being duplicate (Fig. 1). By reviewing the title and abstracts of the remaining articles, 1438 publications not meeting the inclusion criteria were excluded. Subsequently, twenty-three full-text articles were carefully reviewed and eleven clinical trials were excluded because of the following reasons: five studies had a duration of supplementation period of less than 4 weeks; one study enrolled adolescents; two studies involved interventions that were a combination of other components together with L-arginine and the design did not enable evaluating L-arginine effect only; one study enrolled less than ten participants; and two articles reported the results from a same population. Finally, twelve trials ${ }^{(18,20-30)}$ including fifteen treatment arms were considered eligible for the systematic review. However, one of the included articles ${ }^{(28)}$ did not report the data required for the meta-analysis. We contacted the corresponding author of the study twice but did not receive any response, and therefore it was subsequently, excluded, leaving eleven studies $^{(18,20-27,29,30)}$ for inclusion in the meta-analysis.

\section{Study and participant characteristics}

Characteristics of the included trials are outlined in Table 2. In total, 631 participants were enrolled in the selected articles, out of which 359 individuals were allocated to L-arginine supplementation group and 272 subjects to the control group. These studies were published between 1996 and 2019 and were carried

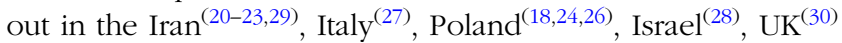
and Germany ${ }^{(25)}$. All studies except two ${ }^{(28,30)}$ adopted a parallel study design. The mean age of the participants ranged from 20.86 to 64.5 years, and the mean baseline BMI varied from 23.67 to $38.35 \mathrm{~kg} / \mathrm{m}^{2}$. Only one ${ }^{(21)}$ of included studies involved exclusively male population, two ${ }^{(28,29)}$ involved women and the other trials involved populations of mixed sex. The follow-up period ranged from 4 to 77 weeks. In these studies, the daily supplementation dosage of L-arginine varied between 1 and $21 \mathrm{~g} / \mathrm{d}$. The health status of the included participants was mixed and included type 2 diabetes patients ${ }^{(22)}$, postmenopausal women ${ }^{(28)}$, patients with $\mathrm{CVD}^{(26)}$, subjects with hypercholesterolaemia $^{(30)}$, obese individuals ${ }^{(18,20,24,29)}$, subjects with hypertriacylglycerolaemia ${ }^{(25)}$, individuals with the metabolic syndrome $^{(23)}$, healthy subjects ${ }^{(21)}$ and those with impaired glucose tolerance and the metabolic syndrome ${ }^{(27)}$. No major adverse effects attributable to intervention or control were reported in RCT.

In a study by Pourghassem Gargari et al. ${ }^{(29)}$, there were three intervention groups (arginine + hypoenergetic diet enriched in legumes (HDEL), arginine $+\mathrm{HDEL}+\mathrm{Se}$ and HDEL + Se) and one control group (HDEL). We considered the result of the arginine + HDEL and HDEL groups as one arm and the result of the arginine + HDEL + Se and HDEL + Se groups as another arm. Furthermore, Rahimi \& Naghizadeh $^{(22)}$ and Dashtabi et al. ${ }^{(20)}$ included two different arginine doses in their trials ( 3 or $6 \mathrm{~g} / \mathrm{d}$ ); therefore, we considered these imputations as four different arms.

\section{Quality assessment}

Among twelve studies included in the present review, nine trials $^{(18,20,21-29)}$ were categorised as good quality and three trials $^{(22,28,30)}$ as fair quality. The details of the risk of bias in individual studies according to the domains used by the Cochrane Collaborations tool are provided in Table 3. 


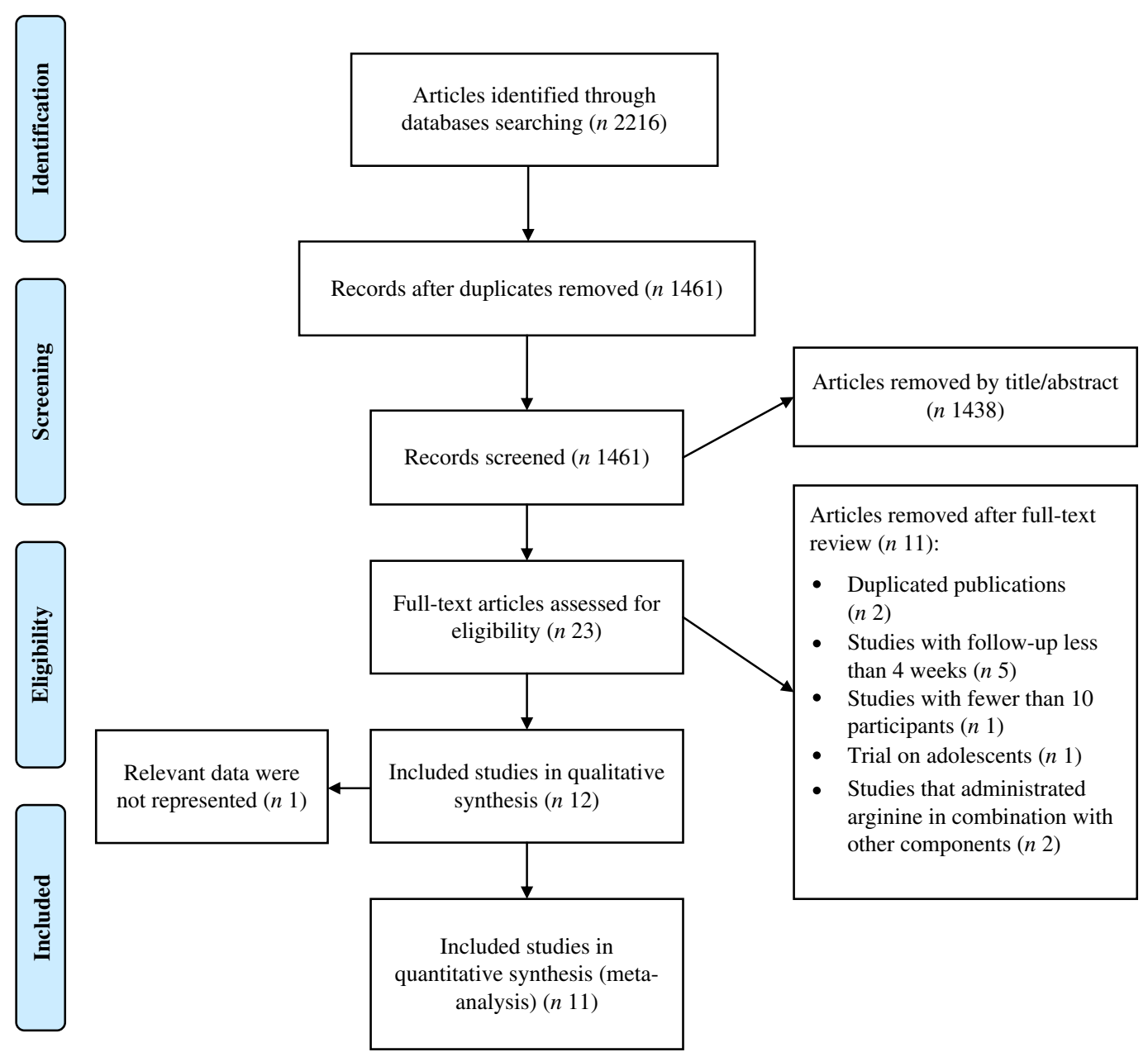

Fig. 1. Preferred reporting items for systematic reviews and meta-analyses (PRISMA) flow diagram of study selection process.

\section{Findings from the systematic review}

The present systematic review revealed that three trials ${ }^{(20-22)}$ reported that L-arginine supplementation managed to reduce TC levels, while eight studies ${ }^{(18,23,25-30)}$ failed to find any significant effect on this parameter. In terms of changes in TAG levels, three trials observed a significant reduction after L-arginine supplementation in references ${ }^{(20,21,23)}$, while eight studies ${ }^{(18,22,25-30)}$ did not find such an effect. Evidence points out that arginine may pose favourable effects on LDL-cholesterol concentration as well - three trials ${ }^{(20-22)}$ found that supplementation induced a decrease in plasma LDL-cholesterol, while other trials report no significant changes in this outcome ${ }^{(23-30)}$. Finally, L-arginine may induce favourable changes in HDL-cholesterol levels as confirmed in two trials; ${ }^{(20,21)}$ however, the remaining studies $^{(22-30)}$ did not reach the same conclusion.

\section{Findings from the meta-analysis}

In total, we pooled the data from thirteen arms corresponding to ten studies ${ }^{(18,20-23,25-27,29,30)}$ which included 561 participants, to estimate the effect of L-arginine supplementation on plasma TC levels. After using a meta-analysis random-effects model, we found that $\mathrm{L}$-arginine supplementation did not significantly affect the serum TC levels (WMD: $-5.03 \mathrm{mg} / \mathrm{dl} ; 95 \% \mathrm{CI}-10.78$, $0.73 ; P=0.08$ ) (Fig. 2). The between-study heterogeneity was non-significant $\left(P=0.07, I^{2}=39.0 \%\right)$. Subgroup analysis based on participants' mean age, baseline BMI, study duration, participants health status and L-arginine dose confirmed that the effect is not statistically significant in any of the subgroups (Table 4). Findings from the sensitivity analysis revealed that the exclusion of any single study from the analysis did not alter the overall effect.

Thirteen arms from ten studies ${ }^{(18,20-23,25-27,29,30)}$ including 561 participants reported the effect of L-arginine on serum TAG concentration. The pooled effect demonstrated a significant decrease in TAG levels following L-arginine supplementation (WMD: $-7.04 \mathrm{mg} / \mathrm{dl} ; 95 \% \mathrm{CI}-11.42,-2.67 ; P<0.001)$ with a non-significant heterogeneity among included studies $\left(P=0.59, I^{2}=0.0 \%\right)$ (Fig. 3). Subgroup analysis based on participants' mean age, baseline BMI, study duration, participants health status and L-arginine dose revealed that the effect was significant in studies that included participants with a mean age $\geq 50$ years (WMD: $-9.08 \mathrm{mg} / \mathrm{dl} ; 95 \%$ CI $-16.70,-1.45$; $P=0.02$ ), a baseline BMI $\geq 30 \mathrm{~kg} / \mathrm{m}^{2}$ (WMD: $-9.86 \mathrm{mg} / \mathrm{dl} ; 95 \%$ CI $-16.22,-3.50 ; P<0.001$ ), L-arginine dose $<6 \mathrm{~g} / \mathrm{d}$ (WMD: $-7.89 \mathrm{mg} / \mathrm{dl} ; 95 \% \mathrm{CI}-15.04,-0.73 ; P=0.03)$, type 2 diabetes/ 
Table 2. Characteristics of included studies

\begin{tabular}{|c|c|c|c|c|c|c|c|c|c|c|c|}
\hline Authors & Country & $\begin{array}{l}\text { Sample } \\
\text { size }\end{array}$ & $\begin{array}{l}\text { RCT design } \\
\text { (blinding) }\end{array}$ & Sex & $\begin{array}{l}\text { Mean age } \\
\text { (years) }\end{array}$ & $\begin{array}{l}\text { Mean BMI } \\
\left(\mathrm{kg} / \mathrm{m}^{2}\right)\end{array}$ & $\begin{array}{l}\text { Duration } \\
\text { (weeks) }\end{array}$ & Target population & $\begin{array}{l}\text { Intervention (name } \\
\text { and daily dose) }\end{array}$ & Control & Results \\
\hline Clarkson et al. ${ }^{(30)}$ & UK & 27 & Crossover (yes) & Both & 29 & 26 & 4 & $\begin{array}{l}\text { Subjects with } \\
\text { hypercholesterolaemia }\end{array}$ & $21 \mathrm{~g} / \mathrm{d}$ arginine & Placebo & $\begin{array}{l}\text { TC } \leftrightarrow \\
\text { TAG } \leftrightarrow \\
\text { LDL-cholesterol } \leftrightarrow \\
\text { HDL-cholesterol } \leftrightarrow\end{array}$ \\
\hline Blum et al. ${ }^{(28)}$ & Israel & 10 & Crossover (yes) & Women & 55 & $26 \cdot 6$ & 4 & Postmenopausal women & $9 \mathrm{~g} / \mathrm{d}$ arginine & Placebo & $\begin{array}{l}\text { TC } \leftrightarrow \\
\text { TAG } \leftrightarrow \\
\text { LDL-cholesterol } \leftrightarrow \\
\text { HDL-cholesterol } \leftrightarrow\end{array}$ \\
\hline Lucotti et al. ${ }^{(26)}$ & Poland & 30 & Parallel (yes) & Both & 64.5 & 34 & 26 & Patients with CVD & $6.4 \mathrm{~g} / \mathrm{d}$ arginine & Placebo & $\begin{array}{l}\text { TC } \leftrightarrow \\
\text { TAG } \leftrightarrow \\
\text { LDL-cholesterol } \leftrightarrow \\
\text { HDL-cholesterol } \leftrightarrow\end{array}$ \\
\hline Schulze et al. (25) & Germany & 33 & Parallel (yes) & Both & $54 \cdot 3$ & $28 \cdot 40$ & 6 & $\begin{array}{l}\text { Subjects with } \\
\text { hypertriacylglycerolaemia }\end{array}$ & $3 \mathrm{~g} / \mathrm{d}$ arginine & Placebo & $\begin{array}{l}\text { TC } \leftrightarrow \\
\text { TAG } \leftrightarrow \\
\text { LDL-cholesterol } \leftrightarrow \\
\text { HDL-cholesterol } \leftrightarrow\end{array}$ \\
\hline $\begin{array}{l}\text { Bogdanski } \\
\text { et al. }{ }^{(24)}\end{array}$ & Poland & 60 & Parallel (yes) & Both & $42 \cdot 4$ & $38 \cdot 35$ & 13 & Obese & $9 \mathrm{~g} / \mathrm{d}$ arginine & Placebo & $\begin{array}{l}\text { LDL-cholesterol } \leftrightarrow \\
\text { HDL-cholesterol } \leftrightarrow\end{array}$ \\
\hline $\begin{array}{l}\text { Rahimi \& } \\
\text { Naghizadeh(22) }\end{array}$ & Iran & 33 & Parallel (yes) & Both & $50 \cdot 89$ & $29 \cdot 07$ & 13 & T2DM & $3 \mathrm{~g} / \mathrm{d}$ arginine & Placebo & $\begin{array}{l}\text { TC } \leftrightarrow \\
\text { TAG } \leftrightarrow \\
\text { LDL-cholesterol } \leftrightarrow \\
\text { HDL-cholesterol } \leftrightarrow\end{array}$ \\
\hline $\begin{array}{l}\text { Rahimi \& } \\
\text { Naghizadeh(22) }\end{array}$ & Iran & 35 & Parallel (yes) & Both & 51.98 & $28 \cdot 55$ & 13 & T2DM & $6 \mathrm{~g} / \mathrm{d}$ arginine & Placebo & $\begin{array}{l}\text { TC } \downarrow \\
\text { TAG } \leftrightarrow \\
\text { LDL-cholesterol } \downarrow \\
\text { HDL-cholesterol } \leftrightarrow\end{array}$ \\
\hline Suliburska et al. ${ }^{(18)}$ & Poland & 88 & Parallel (yes) & Both & $42 \cdot 3$ & $36 \cdot 45$ & 26 & Obese & $9 \mathrm{~g} / \mathrm{d}$ arginine & Placebo & $\begin{array}{l}\mathrm{TC} \leftrightarrow \\
\mathrm{TAG} \leftrightarrow\end{array}$ \\
\hline Pahlavani et al.(21) & Iran & 52 & Parallel (yes) & Male & $20 \cdot 86$ & 23.67 & 7 & Healthy & $2 \mathrm{~g} / \mathrm{d}$ arginine & Placebo & $\begin{array}{l}\text { TC } \downarrow \\
\text { TAG } \downarrow \\
\text { LDL-cholesterol } \downarrow \\
\text { HDL-cholesterol } \uparrow\end{array}$ \\
\hline $\begin{array}{l}\text { Pourghassem } \\
\text { Gargari et al. }{ }^{(29)}\end{array}$ & Iran & 34 & Parallel (yes) & Women & $35 \cdot 2$ & 31.80 & 6 & Obese & $\mathrm{HDEL}+5 \mathrm{~g} / \mathrm{d}$ arginine & HDEL & $\begin{array}{l}\text { TC } \leftrightarrow \\
\text { TAG } \leftrightarrow \\
\text { LDL-cholesterol } \leftrightarrow \\
\text { HDL-cholesterol } \leftrightarrow\end{array}$ \\
\hline $\begin{array}{l}\text { Pourghassem } \\
\text { Gargari et al. }{ }^{(29)}\end{array}$ & Iran & 34 & Parallel (yes) & Women & $35 \cdot 3$ & 32.45 & 6 & Obese & $\begin{array}{l}\mathrm{HDEL}+5 \mathrm{~g} / \mathrm{d} \text { arginine } \\
+200 \mu \mathrm{g} / \mathrm{d} \mathrm{Se}\end{array}$ & $\begin{array}{l}\mathrm{HDEL}+ \\
200 \mu \mathrm{g} / \mathrm{d} \mathrm{Se}\end{array}$ & $\begin{array}{l}\text { TC } \leftrightarrow \\
\text { TAG } \leftrightarrow \\
\text { LDL-cholesterol } \leftrightarrow \\
\text { HDL-cholesterol } \leftrightarrow\end{array}$ \\
\hline Dashtabi et al. ${ }^{(20)}$ & Iran & 41 & Parallel (yes) & Both & $42 \cdot 36$ & 35.44 & 8 & Obese & $3 \mathrm{~g} / \mathrm{d}$ arginine & Placebo & $\begin{array}{l}\text { TC } \downarrow \\
\text { TAG } \leftrightarrow \\
\text { LDL-cholesterol } \downarrow \\
\text { HDL-cholesterol } \leftrightarrow\end{array}$ \\
\hline
\end{tabular}


metabolic syndrome (WMD: $-11.77 \mathrm{mg} / \mathrm{dl} ; 95 \%$ CI -19.39 , $-4 \cdot 14 ; P<0.001$ ) and intervention duration $\geq 10$ weeks (WMD: $-9.21 \mathrm{mg} / \mathrm{dl} ; 95 \% \mathrm{CI}-15 \cdot 20,-3 \cdot 21 ; P<0 \cdot 001$ ) (Table 4 ). The sensitivity analysis demonstrated that by removing the study conducted by Bahrami et al. ${ }^{(23)}$, the effect of L-arginine supplementation on TAG levels becomes non-significant (WMD: $4.65 \mathrm{mg} / \mathrm{dl} ; 95 \% \mathrm{CI}-9 \cdot 51,0 \cdot 19 ; P=0 \cdot 06$ ).

The impact of $\mathrm{L}$-arginine supplementation on LDL-cholesterol levels was assessed in ten trials ${ }^{(20-29,30)}$, with thirteen treatment arms including 533 participants. The meta-analysis revealed that L-arginine supplementation did not significantly affect LDL-cholesterol levels (WMD: $-0.47 \mathrm{mg} / \mathrm{dl} ; 95 \% \mathrm{CI}-3.61$, $2.66 ; P=0.76)$, while the heterogeneity among the included studies was NS $\left(P=0.53, I^{2}=0.0 \%\right)$ (Fig. 4). The subgroup analysis based on participants' mean age, baseline BMI, study duration, participants health status and L-arginine dose also showed that the effect is not statistically significant in any of the subgroups (Table 4). Furthermore, removing each individual study by sensitivity analysis did not change the pooled effect size.

Ten studies ${ }^{(20-27,29,30)}$ including 533 participants from thirteen intervention arms measured changes in serum HDL-cholesterol concentrations following L-arginine supplementation. Pooled results from the random-effects model revealed that L-arginine supplementation had no significant effect on serum HDLcholesterol levels (WMD: $0.57 \mathrm{mg} / \mathrm{dl}$; $95 \%$ CI $-1.28,2.43$; $P=0.54$ ) (Fig. 5). There was a significant heterogeneity among the studies $\left(P<0.001, I^{2}=68.4 \%\right)$, and the subgroup analysis showed that baseline BMI $\left(<30 \mathrm{~kg} / \mathrm{m}^{2} ; P=0.96 ; I^{2}=0.0 \%\right)$, duration of follow-up ( $<10$ weeks: $P=0 \cdot 23, I^{2}=25 \cdot 6 \%$ ), participants health status (dyslipidaemia: $P=0.69, I^{2}=0.0 \%$ ) or (type 2 diabetes/metabolic syndrome: $P=0.93, I^{2}=0.0 \%$ ) and L-arginine dosage ( $<6 \mathrm{~g} / \mathrm{d}: P=0 \cdot 96 ; I^{2}=0.0 \%$ ) were significant contributors to the between-study heterogeneity. Besides, the subgroup analysis showed that L-arginine supplementation increases HDL levels in trials with a follow-up duration $<10$ weeks (WMD: $2.04 \mathrm{mg} / \mathrm{dl}$; $95 \%$ CI $0.52,3.56 ; P=0.01$ ) (Table 4). Findings from the sensitivity analysis revealed that the exclusion of any single study from the analysis did not alter the overall effect.

\section{Publication bias}

Although the visual inspection of funnel plots showed slight asymmetries, no significant publication bias was detected in the meta-analyses in the case of TC (Begg's test, $P=0.54$; Egger's test, $P=0.45$ ), TAG (Begg's test, $P=0.54$; Egger's test, $P=0.56$ ), LDL-cholesterol (Begg's test, $P=0 \cdot 39$; Egger's test, $P=0 \cdot 80$ ) or HDL-cholesterol (Begg's test, $P=0 \cdot 39$; Egger's test, $P=0 \cdot 86)$.

\section{Discussion}

To the best of our knowledge, the present study is the first systematic review and meta-analysis that measured the effect of L-arginine supplementation on lipid profile by summarising the data from published RCT. Our results indicate that L-arginine supplementation was not able to induce changes in TC, 
Table 3. Quality assessment of included studies based on Cochrane guidelines

\begin{tabular}{|c|c|c|c|c|c|c|c|c|}
\hline Study & $\begin{array}{l}\text { Random } \\
\text { sequence } \\
\text { generation }\end{array}$ & $\begin{array}{l}\text { Allocation } \\
\text { concealment }\end{array}$ & $\begin{array}{l}\text { Blinding of } \\
\text { participants, } \\
\text { personnel }\end{array}$ & $\begin{array}{l}\text { Blinding of } \\
\text { outcome } \\
\text { assessment }\end{array}$ & $\begin{array}{l}\text { Incomplete } \\
\text { outcome } \\
\text { data }\end{array}$ & $\begin{array}{l}\text { Selective } \\
\text { outcome } \\
\text { reporting }\end{array}$ & $\begin{array}{l}\text { Other } \\
\text { sources } \\
\text { of bias }\end{array}$ & $\begin{array}{l}\text { Overall } \\
\text { quality }\end{array}$ \\
\hline Clarkson et al. ${ }^{(30)}$ & Low & Unclear & Low & Unclear & Low & Unclear & Unclear & Fair \\
\hline Blum et al. ${ }^{(28)}$ & Low & Unclear & Low & Unclear & Unclear & Low & Unclear & Fair \\
\hline Lucotti et al. ${ }^{(26)}$ & Low & Low & Low & Low & Low & Low & Low & Good \\
\hline Schulze et al. (25) & Low & Low & Low & Unclear & Low & Low & Unclear & Good \\
\hline Bogdanski et al. ${ }^{(24)}$ & Low & Low & Low & Unclear & Low & Low & Low & Good \\
\hline Rahimi \& Naghizadeh ${ }^{(22)}$ & Low & Unclear & Low & Unclear & Low & Unclear & Unclear & Fair \\
\hline Suliburska et al. ${ }^{(18)}$ & Low & Low & Low & Unclear & Low & Low & Low & Good \\
\hline Pahlavani et al.(21) & Low & Low & Low & Low & Low & Low & Low & Good \\
\hline Pourghassem Gargari et al. ${ }^{(29)}$ & Low & Low & Low & Low & Low & Low & Low & Good \\
\hline Dashtabi et al. (20) & Low & Low & Low & Unclear & Low & Low & Unclear & Good \\
\hline Monti et al. ${ }^{(27)}$ & Low & Low & Low & Unclear & Low & Low & Low & Good \\
\hline Bahrami et al. ${ }^{(23)}$ & Low & Low & Low & Unclear & Low & Low & Low & Good \\
\hline
\end{tabular}

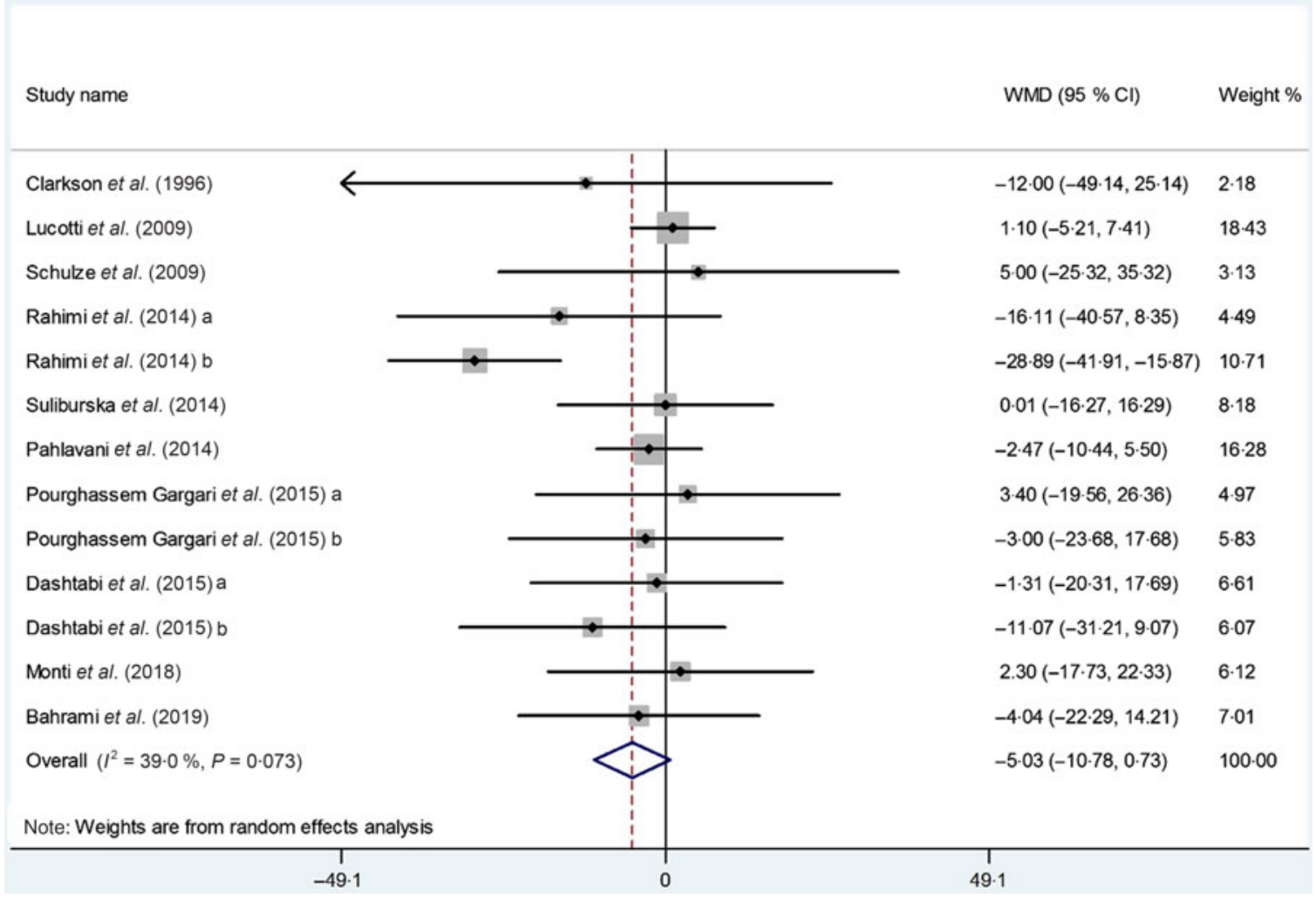

Fig. 2. Forest plot of the effect of L-arginine supplementation on total cholesterol. WMD, weighted mean difference.

LDL-cholesterol and HDL-cholesterol concentrations; however, it did induce a significant decrease in TAG levels. Subgroup analyses further confirmed that L-arginine supplementation imposed a significant TAG-lowering effect in studies that implemented long-term treatment ( $\geq 10$ weeks) as well as in studies where participants' baseline BMI was $\geq 30 \mathrm{~kg} / \mathrm{m}^{2}$, mean age was $\geq 50$ years, supplementation dosage was $<6 \mathrm{~g} / \mathrm{d}$ and participants were type 2 diabetes or metabolic syndrome patients. Although the pooled effect size of L-arginine supplementation on HDL-cholesterol levels was NS, subgroup analysis revealed that this effect was significant only in studies that lasted longer than 10 weeks.
In this meta-analysis, we concluded that L-arginine was able to induce favourable changes only on TAG levels, while this was not observed in the case of other lipid parameters. The possible explanation for the observed lack of significant effect for all parameters except TAG might be the fact that the included population had only TAG levels above the recommended upper value, according to the definition of the metabolic syndrome. Thus, this observation may imply that individuals with higher TAG levels are better respondents to L-arginine therapy.

The biological plausibility of lipid-associated L-arginine implications comes from the existing relationship between this amino acid and glucose metabolism. Human clinical trials have 


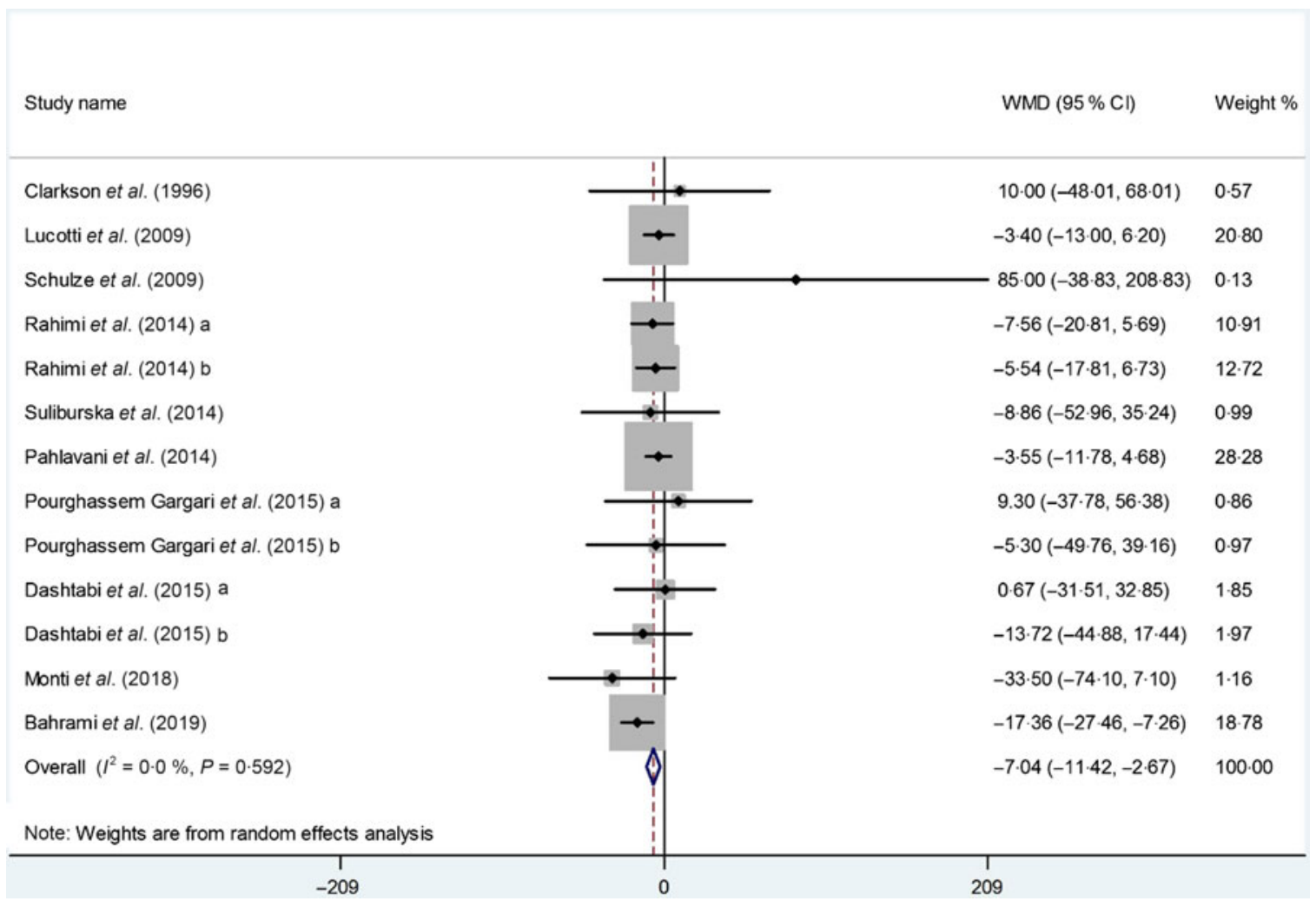

Fig. 3. Forest plot of the effect of L-arginine supplementation on TAG. WMD, weighted mean difference.

concluded that L-arginine can be an effective tool in reducing blood glucose levels in type 2 diabetes patients ${ }^{(36)}$, increasing insulin sensitivity ${ }^{(37)}$ and improving insulin resistance ${ }^{(38)}$. One of the proposed explanations for the observed results is that insulin resistance of the adipocytes can lead to an increased release of fatty acids into the circulation. Increased free fatty acid flux reaches the liver where it stimulates the assembly and secretion of VLDL, which results in hypertriacylglycerolaemia ${ }^{(38,39)}$. Another possible mechanism might be related to the lowering effect of L-arginine on blood glucose, the decrease in blood glucose levels leads to an increase in the concentration of cyclic AMP which in turn decreases the TAG levels ${ }^{(40,41)}$. Therefore, given the beneficial role of $\mathrm{L}$-arginine in the glucose homeostasis, it is proposed that supplementation with this amino acid can lower serum TAG levels ${ }^{(41,42)}$.

Other putative mechanisms may be related to the arginine NO synthase pathway. All NO synthase isoforms utilise L-arginine as a substrate, which undergoes a two-step metabolic conversion, yielding at the end $\mathrm{L}$-citrulline and $\mathrm{NO}^{(43)}$. Elevated NO production consequently increases lipoprotein lipase activity $^{(44)}$; and finally by performing hydrolysis of TAG, NO reduces TAG concentration in the plasma ${ }^{(25,45)}$. Animal study data also confirm L-arginine to be an effective lipid-lowering agent by decreasing the white fat expansion and improving serum TAG levels in rats ${ }^{(40,46)}$

A neutral effect of L-arginine supplementation on TC, LDLcholesterol and HDL-cholesterol was observed in the present meta-analysis. Subgroup analysis of the effect of L-arginine supplementation on cholesterol or lipoprotein levels did not moderate the outcome. These findings are generally in line with the majority of individual studies selected for this review. Only three studies ${ }^{(20-22)}$ showed a significant change in TC, LDLcholesterol and HDL-cholesterol levels and others failed to find such a relationship. Furthermore, not all animal studies reported consistent results; as reducing ${ }^{(47,48)}$ or even increasing effect of L-arginine supplementation on cholesterol level has been reported $^{(49)}$. Madeira et al. reported that dietary L-arginine supplementation increases the concentration of total lipids, VLDL and $\mathrm{TAG}^{(50)}$. However, Hu et al $^{(51)}$ reported that L-arginine supplementation decreases TAG and cholesterol levels in the plasma. In addition, He et al. ${ }^{(52)}$ showed that L-arginine supplementation reduces VLDL, lipids and TAG concentrations in piglets. However, some animal studies reported that L-arginine might reduce cholesterol or lipoprotein levels by these mechanisms: (1) decrease the expression of hepatic 3-hydroxyl-3methylglutaryl-CoA reductase mRNA, which shows interaction of L-arginine with cholesterol metabolism ${ }^{(53)}$; (2) increased the lipolysis as well as the oxidation of fatty acids and (3) increase plasma adiponectin levels which improved NEFA $\beta$-oxidation. On the other hand, others reported that possible TC increasing effect of L-arginine could be due to increased fat accretion in the carcass $^{(54)}$. L-Arginine can also increase the level of HDLcholesterol through its effect on inflammation ${ }^{(46,55,56)}$.

L-Arginine has generally been well tolerated when administered in small doses $(\leq 30 \mathrm{~g} / \mathrm{d})^{(57)}$. However, there were some reported benign side effects, which include abdominal pain, bloating, nausea and vomiting, airway inflammation, diarrhoea, hypotension, worsening of asthma and allergic reactions ${ }^{(57-59)}$. 
Table 4. Result of subgroup analysis of included studies in meta-analysis

(Effect sizes and $95 \%$ confidence intervals; inconsistency indices $\left(I^{2}\right)$; $P$ values)

\begin{tabular}{|c|c|c|c|c|c|c|}
\hline Subgrouped by & No. of trials & Effect size ${ }^{*}$ & $95 \% \mathrm{Cl}$ & $I^{2}(\%)$ & $P$ for heterogeneity & $P$ for effect size \\
\hline \multicolumn{7}{|l|}{ TC } \\
\hline \multicolumn{7}{|l|}{ Mean age (years) } \\
\hline$\geq 50$ & 6 & -7.47 & $-19.97,5.04$ & 72.5 & $<0.001$ & 0.24 \\
\hline$<50$ & 7 & $-2 \cdot 66$ & $-8.46,3.14$ & 0.0 & 0.97 & 0.41 \\
\hline \multicolumn{7}{|c|}{ Baseline BMI $\left(\mathrm{kg} / \mathrm{m}^{2}\right)$} \\
\hline$\geq 30$ & 8 & -0.23 & $-5.01,4.54$ & 0.0 & 0.97 & 0.92 \\
\hline$<30$ & 5 & -11.95 & $-26 \cdot 64,2 \cdot 75$ & 68.5 & 0.01 & $0 \cdot 16$ \\
\hline \multicolumn{7}{|c|}{ Intervention duration (weeks) } \\
\hline$\geq 10$ & 6 & -7.38 & $-18.78,4.02$ & $72 \cdot 4$ & $<0.001$ & 0.20 \\
\hline$<10$ & 7 & $-2 \cdot 72$ & $-8.81,3.36$ & 0.0 & 0.96 & 0.43 \\
\hline \multicolumn{7}{|l|}{ Dose $(g / d)$} \\
\hline$\geq 6$ & 6 & $-7 \cdot 74$ & $-19 \cdot 74,4 \cdot 25$ & 71.9 & $<0.001$ & 0.26 \\
\hline$<6$ & 7 & -2.69 & $-8.61,3.23$ & 0.0 & 0.94 & 0.37 \\
\hline \multicolumn{7}{|l|}{ Health status } \\
\hline Dyslipidaemia & 2 & -1.80 & $-25 \cdot 28,21.69$ & 0.0 & 0.48 & 0.88 \\
\hline T2D/MetS & 4 & -12.58 & $-28 \cdot 19,3.02$ & $65 \cdot 2$ & 0.03 & $0 \cdot 11$ \\
\hline Other & 7 & -0.79 & $-5.09,3.51$ & 0.0 & 0.94 & 0.71 \\
\hline \multicolumn{7}{|l|}{ TAG } \\
\hline \multicolumn{7}{|l|}{ Mean age (years) } \\
\hline$\geq 50$ & 6 & -9.08 & $-16 \cdot 70,-1 \cdot 45$ & 36.7 & 0.16 & 0.02 \\
\hline$<50$ & 7 & -3.56 & $-10.91,3.79$ & 0.0 & 0.98 & 0.31 \\
\hline \multicolumn{7}{|c|}{ Baseline BMI $\left(\mathrm{kg} / \mathrm{m}^{2}\right)$} \\
\hline$\geq 30$ & 8 & -9.86 & $-16 \cdot 22,-3 \cdot 50$ & 0.0 & 0.50 & $<0.001$ \\
\hline$<30$ & 5 & -4.51 & $-10.54,1.53$ & 0.0 & 0.63 & 0.13 \\
\hline \multicolumn{7}{|c|}{ Intervention duration (weeks) } \\
\hline$\geq 10$ & 6 & $-9 \cdot 21$ & $-15 \cdot 20,-3 \cdot 21$ & 12.0 & 0.33 & $<0.001$ \\
\hline$<10$ & 7 & -3.09 & $-10.53,4.35$ & 0.0 & 0.81 & 0.38 \\
\hline \multicolumn{7}{|l|}{ Dose $(g / d)$} \\
\hline$\geq 6$ & 6 & $-5 \cdot 50$ & $-12.58,1.58$ & 0.0 & 0.76 & $0 \cdot 11$ \\
\hline$<6$ & 7 & -7.89 & $-15.04,-0.73$ & 18.9 & 0.28 & 0.03 \\
\hline Health status & & & & & & \\
\hline Dyslipidaemia & 2 & $26 \cdot 73$ & $-34.47,87.93$ & 13.5 & 0.28 & 0.39 \\
\hline T2D/MetS & 4 & -11.77 & $-19 \cdot 39,-4 \cdot 14$ & 17.9 & 0.30 & $<0.001$ \\
\hline Other & 7 & -3.46 & $-9.50,2.23$ & 0.0 & 0.99 & 0.22 \\
\hline LDL-cholesterol & & & & & & \\
\hline Mean age (years & & & & & & \\
\hline$\geq 50$ & 6 & -0.44 & $-7 \cdot 23,6 \cdot 34$ & $42 \cdot 1$ & 0.12 & 0.89 \\
\hline$<50$ & 7 & -1.69 & $-6 \cdot 31,2.93$ & 0.0 & 0.94 & 0.44 \\
\hline Baseline BMI $(\mathrm{k}$ & & & & & & \\
\hline$\geq 30$ & 8 & $2 \cdot 39$ & $-1 \cdot 93,6 \cdot 71$ & 0.0 & 0.75 & 0.21 \\
\hline$<30$ & 5 & -3.66 & $-8.22,0.90$ & 0.0 & 0.53 & $0 \cdot 10$ \\
\hline Intervention dura & & & & & & \\
\hline$\geq 10$ & 6 & -0.17 & $-6 \cdot 58,6 \cdot 24$ & 42.5 & 0.12 & 0.95 \\
\hline$<10$ & 7 & -2.00 & $-6 \cdot 75,2 \cdot 76$ & 0.0 & 0.96 & 0.38 \\
\hline Dose $(g / d)$ & & & & & & \\
\hline$\geq 6$ & 6 & -0.48 & $-7 \cdot 82,6 \cdot 87$ & $47 \cdot 1$ & 0.09 & 0.85 \\
\hline$<6$ & 7 & -1.65 & $-6 \cdot 21,2 \cdot 91$ & 0.0 & 0.98 & 0.47 \\
\hline Health status & & & & & & \\
\hline Dyslipidaemia & 2 & $6 \cdot 1$ & $-17.79,29.79$ & 0.0 & 0.99 & 0.62 \\
\hline T2D/MetS & 4 & -4.47 & $-11 \cdot 68,2 \cdot 73$ & 13.8 & 0.32 & 0.22 \\
\hline Other & 7 & 0.82 & $-2 \cdot 81,4.44$ & 0.0 & 0.58 & 0.66 \\
\hline HDL-cholesterol & & & & & & \\
\hline Mean age (years & & & & & & \\
\hline$\geq 50$ & 6 & -0.15 & $-2 \cdot 77,2 \cdot 47$ & 51.0 & 0.07 & 0.90 \\
\hline$<50$ & 7 & 1.29 & $-0 \cdot 62,3 \cdot 21$ & 58.0 & 0.05 & 0.21 \\
\hline Baseline BMI (kc & & & & & & \\
\hline$\geq 30$ & 8 & 0.36 & $-2.03,2.75$ & $81 \cdot 2$ & $<0.001$ & 0.76 \\
\hline$<30$ & 5 & 1.24 & $-1.51,3.99$ & 0.0 & 0.96 & 0.36 \\
\hline Intervention dura & & & & & & \\
\hline$\geq 10$ & 6 & -0.85 & $-3.28,1.58$ & 48.9 & 0.08 & 0.49 \\
\hline$<10$ & 7 & 2.04 & $0.52,3.56$ & 25.6 & 0.23 & 0.01 \\
\hline Dose $(g / d)$ & & & & & & \\
\hline$\geq 6$ & 6 & -0.25 & $-4 \cdot 36,3 \cdot 87$ & 86.0 & $<0.001$ & 0.90 \\
\hline$<6$ & 7 & $1 \cdot 18$ & $-0.16,2.52$ & 0.0 & 0.96 & 0.08 \\
\hline Health status & & & & & & \\
\hline Dyslipidaemia & 2 & 1.88 & $-5 \cdot 26,9.02$ & 0.0 & 0.69 & 0.60 \\
\hline T2D/MetS & 4 & 1.25 & $-1.09,3.59$ & 0.0 & 0.93 & 0.29 \\
\hline Other & 7 & 0.21 & $-2 \cdot 42,2 \cdot 85$ & 83.8 & $<0.001$ & 0.87 \\
\hline
\end{tabular}

TC, total cholesterol; T2D, type 2 diabetes mellitus; MetS, metabolic syndrome.

*Calculated by random-effects model. 
Clarkson et al. (1996)

Lucotti et al. (2009)

Schulze et al. (2009)

Bogdanski et al. (2012)

Rahimi et al. (2014) a

Rahimi et al. (2014) b

Pahlavani et al. (2014)

Pourghassem Gargari et al. (2015) a

Pourghassem Gargari et al. (2015) b

Dashtabi et al. (2015) a

Dashtabi et al. (2015) b

Monti et al. (2018)

Bahrami et al. (2019)

Overall $\left(I^{2}=0.0 \%, P=0.539\right)$

Note: Weights are from random effects analysis $-41 \cdot 3$

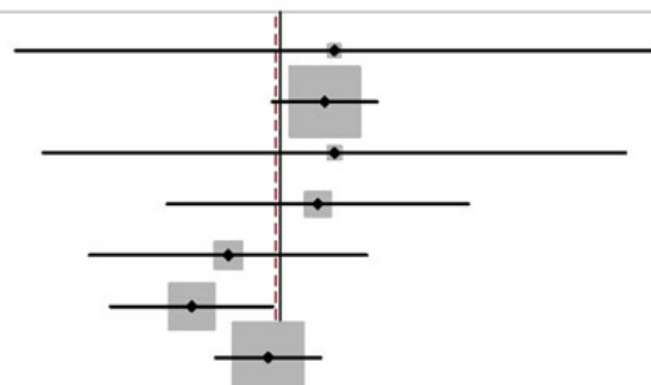

$6.00(-29 \cdot 26,41 \cdot 26) \quad 0.79$

$4.90(-0.89,10.69) \quad 29.31$

$6.00(-26 \cdot 22,38.22) \quad 0.95$

$4 \cdot 13(-12 \cdot 53,20 \cdot 79) \quad 3.54$

$-5.74(-21 \cdot 10,9.62) \quad 4.17$

$-9.81(-18 \cdot 76,-0.86) \quad 12 \cdot 28$

$-1.35(-7 \cdot 16,4 \cdot 46) \quad 29 \cdot 18$

$1.20(-21.55,23.95) \quad 1.90$

$-1.00(-19.48,17 \cdot 48) \quad 2.88$

$-5.88(-21.64,9.88) \quad 3.96$

$-8.75(-24 \cdot 64,7 \cdot 14) \quad 3.90$

$7 \cdot 60(-10 \cdot 84,26 \cdot 04) \quad 2 \cdot 89$

$0.85(-14 \cdot 37,16 \cdot 07) \quad 4.24$

$-0.47(-3 \cdot 61,2 \cdot 66) \quad 100 \cdot 00$

Fig. 4. Forest plot of the effect of L-arginine supplementation on LDL-cholesterol. WMD, weighted mean difference.

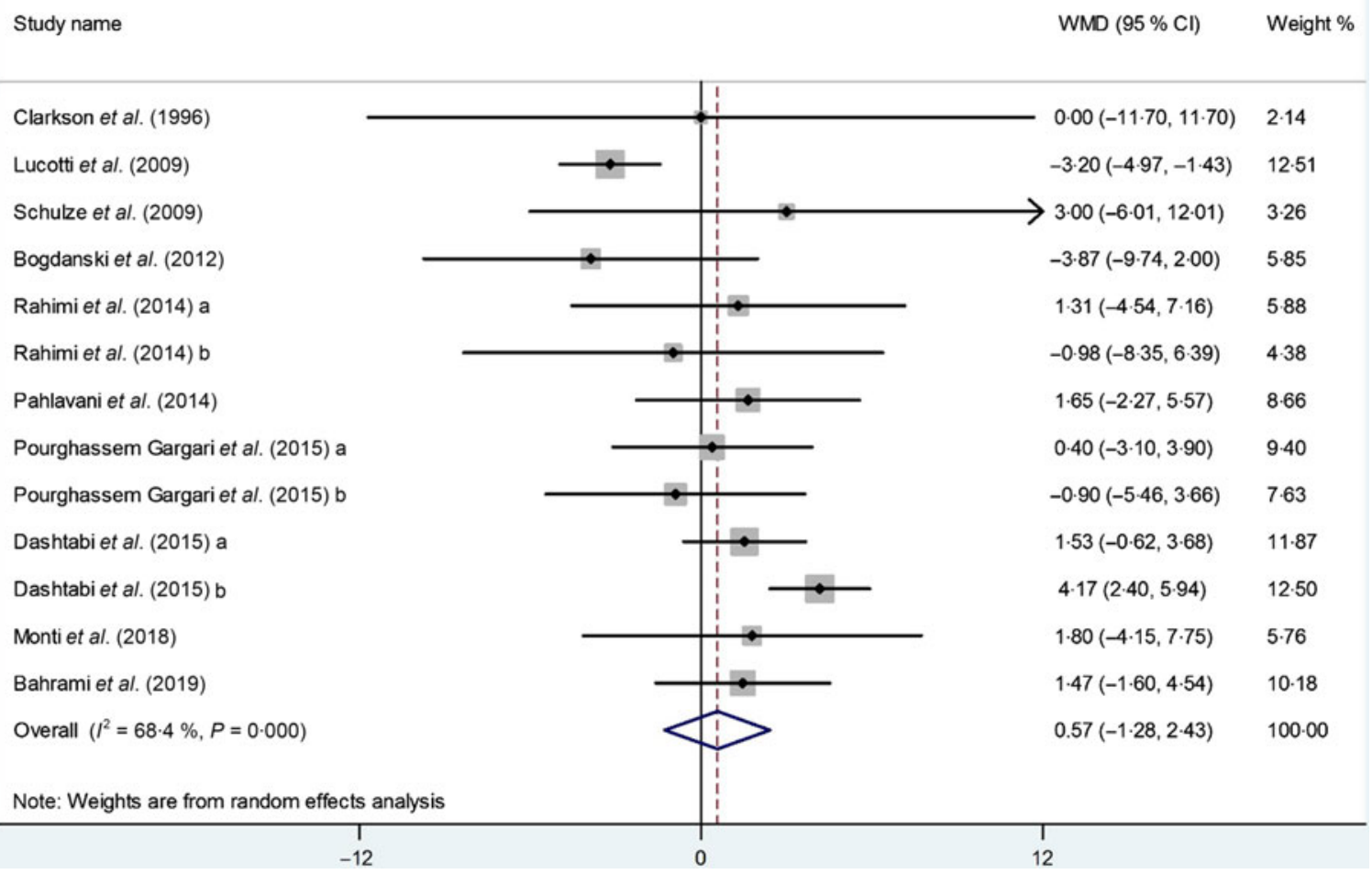

Fig. 5. Forest plot of the effect of L-arginine supplementation on HDL-cholesterol. WMD, weighted mean difference. 
Furthermore, given that a major part of L-arginine is metabolised to ornithine and urea ${ }^{(57,60)}$, patients with gout or renal function impairment should pay special caution when consuming it. Finally, due to its vasodilating properties, L-arginine has also been shown to interfere with certain medications (including Viagra and blood pressure medications) thus imposing negative reactions ${ }^{(57)}$.

This meta-analysis has certain limitations that should be noted. First, the included studies involved individuals with different health status, resulting in a heterogeneous sample. Second, the sample sizes of individual trials were small, thus our results might more easily suffer from sample imbalances and an influence of baseline confounding factors. Third, the influence of sex remains unknown, since there was just one article that involved men. Women have different sex hormones compared with men that may affect lipid profile, thus implying that L-arginine may impose sex-dependent effects. Lastly, most RCT were not primarily designed to assess the effects of L-arginine on lipid concentrations. In order to draw straightforward conclusions regarding recommending L-arginine supplementation as a lipid-lowering agent, we need more RCT designed to specifically address this issue in a target population of patients with abnormal lipid profile.

The present study also has its strengths. It is only a systematic review and meta-analysis study to investigate the effect of arginine supplementation on lipid profile. Our systematic search makes it unlikely that large reports were missed, and error and bias were minimised by independent, duplicate decisions on the whole process of review by adhering to the PRISMA guidelines. Also, subgroup analysis and assessment of mean age, baseline BMI, dose of L-arginine, study duration and health status were done on the overall effect sizes.

\section{Conclusion}

The present systematic review and meta-analysis demonstrated that L-arginine supplementation leads to a significant reduction in TAG levels. However, no significant effect was observed in the case of other lipid parameters including TC, LDLcholesterol and HDL-cholesterol. In order to confirm the results of our study, further clinical trials that exclusively examine the effects of L-arginine on participants with dyslipidaemia are required.

\section{Acknowledgements}

This research did not receive any specific grant from funding agencies in the public, commercial or not-for-profit sectors.

A. H. and E. G. wrote the concept, design and carried out drafting of the present study. A. A. and E. G. performed searches of the electronic databases, screened the articles and extracted the data. A. H. performed the acquisition, analysis and interpretation of data. A. H. and E. G. critically revised the manuscript. A. P. performed a final revision and proofread of the article. All authors approved the final version of the manuscript. A. H. and E. G. are the guarantors of the present study.

The authors declare no conflict of interest.

\section{References}

1. Yusuf S, Reddy S, Ônpuu S, et al. (2001) Global burden of cardiovascular diseases: Part II: variations in cardiovascular disease by specific ethnic groups and geographic regions and prevention strategies. Circulation 104, 2855-2864.

2. Leal J, Luengo-Fernández R, Gray A, et al. (2006) Economic burden of cardiovascular diseases in the enlarged European Union. Eur Heart J 27, 1610-1619.

3. Mozaffarian D, Benjamin EJ, Go AS, et al. (2016) Heart disease and stroke statistics - 2016 update: a report from the American Heart Association. Circulation 133, e38-e360.

4. Rader DJ (2007) Effect of insulin resistance, dyslipidemia, and intra-abdominal adiposity on the development of cardiovascular disease and diabetes mellitus. Am J Med 120, S12-S18.

5. Lehto S, Rönnemaa T, Haffher SM, et al. (1997) Dyslipidemia and hyperglycemia predict coronary heart disease events in middle-aged patients with NIDDM. Diabetes 46, 1354-1359.

6. McPherson R, Frohlich J, Fodor G, et al. (2006) Canadian Cardiovascular Society position statement - recommendations for the diagnosis and treatment of dyslipidemia and prevention of cardiovascular disease. Can J Cardiol 22, 913-927.

7. Ito MK (2012) Dyslipidemia: management using optimal lipidlowering therapy. Ann Pharmacother 46, 1368-1381.

8. Chang Y \& Robidoux J (2017) Dyslipidemia management update. Curr Opin Pharmacol 33, 47-55.

9. Björnsson ES (2017) Hepatotoxicity of statins and other lipidlowering agents. Liver Int 37, 173-178.

10. Padala S \& Thompson PD (2012) Statins as a possible cause of inflammatory and necrotizing myopathies. Atherosclerosis 222, 15-21.

11. Chalasani $\mathrm{N}$ (2005) Statins and hepatotoxicity: focus on patients with fatty liver. Hepatology 41, 690-695.

12. McNeal CJ, Meininger CJ, Reddy D, et al. (2016) Safety and effectiveness of arginine in adults. J Nutr 146, 2587S-2593S.

13. Ástvaldsdóttir Á, Naimi-Akbar A, Davidson T, et al. (2016) Arginine and caries prevention: a systematic review. Caries Res 50, 383-393.

14. McRae MP (2016) Therapeutic benefits of L-arginine: an umbrella review of meta-analyses. J Chiropractic Med 15, 184-189.

15. Stechmiller JK, Childress B \& Cowan L (2005) Arginine supplementation and wound healing. Nutr Clin Prac 20, 52-61.

16. Popovic PJ, Zeh III HJ \& Ochoa JB (2007) Arginine and immunity. J Nutr 137, 1681S-1686S.

17. Rodrigues-Krause J, Krause M, Rocha I, et al. (2019) Association of L-arginine supplementation with markers of endothelial function in patients with cardiovascular or metabolic disorders: a systematic review and meta-analysis. Nutrients 11, 15.

18. Suliburska J, Bogdanski P, Szulinska M, et al. (2014) Changes in mineral status are associated with improvements in insulin sensitivity in obese patients following L-arginine supplementation. Eur J Nutr 53, 387-393.

19. McRae MP (2016) Therapeutic benefits of L-arginine: an umbrella review of meta-analyses. J Chiropr Med 15, 184-189.

20. Dashtabi A, Mazloom Z, Fararouei M, et al. (2015) Oral L-arginine administration improves anthropometric and biochemical indices associated with cardiovascular diseases in obese patients: a randomized, single blind placebo controlled clinical trial. Res Cardiovasc Med 5, e29419.

21. Pahlavani N, Jafari M, Sadeghi O, et al. (2014) L-Arginine supplementation and risk factors of cardiovascular diseases in healthy men: a double-blind randomized clinical trial. F1000Res 3, 306.

22. Rahimi M \& Naghizadeh MM (2014) The effect of L-arginin supplementation on lipid profiles in patients with diabetes type 2 . J Fasa Univ Med Sci 4, 99-110. 
23. Bahrami D, Mozaffari-Khosravi H \& Zavar-Reza J (2019) The effect of oral L-arginine supplementation on lipid profile, glycemic status, and insulin resistance in patients with metabolic syndrome: a randomized, double-blind, placebo-controlled trial. Mediterranean J Nutr Metab 12, 79-90.

24. Bogdanski P, Suliburska J, Grabanska K, et al. (2012) Effect of 3-month $\mathrm{L}$-arginine supplementation on insulin resistance and tumor necrosis factor activity in patients with visceral obesity. Eur Rev Med Pharmacol Sci 16, 816-823.

25. Schulze F, Glos S, Petruschka D, et al. (2009) L-Arginine enhances the triglyceride-lowering effect of simvastatin in patients with elevated plasma triglycerides. Nutr Res 29, 291-297.

26. Lucotti P, Monti L, Setola E, et al. (2009) Oral L-arginine supplementation improves endothelial function and ameliorates insulin sensitivity and inflammation in cardiopathic nondiabetic patients after an aortocoronary bypass. Metabolism $\mathbf{5 8}$, 1270-1276.

27. Monti LD, Galluccio E, Villa V, et al. (2018) Decreased diabetes risk over 9 year after 18-month oral L-arginine treatment in middle-aged subjects with impaired glucose tolerance and metabolic syndrome (extension evaluation of L-arginine study). Eur J Nutr 57, 2805-2817.

28. Blum A, Cannon III RO, Costello R, et al. (2000) Endocrine and lipid effects of oral L-arginine treatment in healthy postmenopausal women. J Lab Clin Med 135, 231-237.

29. Pourghassem Gargari B, Alizadeh M, Safaeiyan A, et al. (2015) Effect of L-arginine and selenium on metabolic features, insulin resistance and hepatic function tests in obese women. Curr Nutr Food Sci 11, 93-98.

30. Clarkson P, Adams MR, Powe AJ, et al. (1996) Oral L-arginine improves endothelium-dependent dilation in hypercholesterolemic young adults. J Clin Invest 97, 1989-1994.

31. Moher D, Liberati A, Tetzlaff J, et al. (2009) Preferred reporting items for systematic reviews and meta-analyses: the PRISMA statement. Ann Internal Med 151, 264-269.

32. Higgins J \& Green S (2014) Cochrane Handbook for Systematic Reviews of Interventions. Version 5.1.0. The Cochrane Collaboration. Chichester: John Wiley \& Sons Ltd.

33. DerSimonian R \& Laird N (1986) Meta-analysis in clinical trials. Control Clin Trials 7, 177-188.

34. Begg CB \& Mazumdar M (1994) Operating characteristics of a rank correlation test for publication bias. Biometrics $\mathbf{5 0}$, 1088-1101.

35. Egger M, Smith GD, Schneider M, et al. (1997) Bias in meta-analysis detected by a simple, graphical test. BMJ $\mathbf{3 1 5}$, 629-634.

36. Wascher TC, Graier WF, Dittrich P, et al. (1997) Effects of lowdose L-arginine on insulin-mediated vasodilatation and insulin sensitivity. Eur J Clin Invest 27, 690-695.

37. Natarajan Sulochana K, Lakshmi S, Punitham R, et al. (2002) Effect of oral supplementation of free amino acids in type 2 diabetic patients - a pilot clinical trial. Med Sci Monit 8, Cr131-Cr137.

38. Grundy SM (1999) Hypertriglyceridemia, insulin resistance, and the metabolic syndrome. Am J Cardiol 83, 25-29.

39. Ginsberg HN (2000) Insulin resistance and cardiovascular disease. J Clin Invest 106, 453-458.

40. Wu Z, Satterfield MC, Bazer FW, et al. (2012) Regulation of brown adipose tissue development and white fat reduction by L-arginine. Curr Opin Clin Nutr Metab Care 15, 529-538.
41. Paolisso G, Tagliamonte MR, Marfella R, et al. (1997) L-Arginine but not D-arginine stimulates insulin-mediated glucose uptake. Metabolism 46, 1068-1073.

42. Li X, Bazer FW, Gao H, et al. (2009) Amino acids and gaseous signaling. Amino Acids 37, 65-78.

43. Forstermann U \& Sessa WC (2012) Nitric oxide synthases: regulation and function. Eur Heart J 33, 829-837, 837a-837d.

44. Ricart-Jane D, Casanovas A, Jane N, et al. (2008) Nitric oxide and the release of lipoprotein lipase from white adipose tissue. Cell Physiol Biochem 22, 525-530.

45. Mendez J \& Balderas F (2001) Regulation of hyperglycemia and dyslipidemia by exogenous $\mathrm{L}$-arginine in diabetic rats. Biochimie 83, 453-458.

46. de Castro Barbosa T, Jiang LQ, Zierath JR, et al. (2013) L-Arginine enhances glucose and lipid metabolism in rat L6 myotubes via the NO/c-GMP pathway. Metabolism 62, 79-89.

47. El-Kirsh AAA, Abd El-Wahab HMF \& Abd-Ellah Sayed HF (2011) The effect of L-arginine or L-citrulline supplementation on biochemical parameters and the vascular aortic wall in high-fat and high-cholesterol-fed rats. Cell Biochem Func 29, 414-428.

48. Emadi M, Jahanshiri F, Kaveh K, et al. (2011) Nutrition and immunity: the effects of the combination of arginine and tryptophan on growth performance, serum parameters and immune response in broiler chickens challenged with infectious bursal disease vaccine. Avian Pathol 40, 63-72.

49. Kumar P, Kumar A \& Tiwari S (2005) L-Arginine supplementation increases serum cholesterol level. Indian J Pharmacol 37, 183.

50. dos Santos Madeira MSM, Rolo ESA, Pires VMR, et al. (2017) Arginine supplementation modulates pig plasma lipids, but not hepatic fatty acids, depending on dietary protein level with or without leucine. BMC Vet Res 13, 145.

51. Hu S, Li X, Rezaei R, et al. (2015) Safety of long-term dietary supplementation with L-arginine in pigs. Amino Acids 47, 925-936.

52. He Q, Kong X, Wu G, et al. (2009) Metabolomic analysis of the response of growing pigs to dietary L-arginine supplementation. Amino Acids 37, 199.

53. Fouad A, El-Senousey H, Yang X, et al. (2013) Dietary L-arginine supplementation reduces abdominal fat content by modulating lipid metabolism in broiler chickens. Animal 7, 1239-1245.

54. Madeira M, Alfaia C, Costa P, et al. (2014) The combination of arginine and leucine supplementation of reduced crude protein diets for boars increases eating quality of pork. J Anim Sci $\mathbf{9 2}$, 2030-2040

55. Tan B, Yin Y, Liu Z, et al. (2011) Dietary L-arginine supplementation differentially regulates expression of lipid-metabolic genes in porcine adipose tissue and skeletal muscle. J Nutr Biochem 22, 441-445.

56. Harisa G (2011) L-Arginine ameliorates arylesterase/paraoxonase activity of paraoxonase 1 in hypercholesterolemic rats. Asian J Biochem 6, 263-272.

57. Böger RH \& Bode-Böger SM (2001) The clinical pharmacology of L-arginine. Ann Rev Pharmacol Toxicol 41, 79-99.

58. Böger RH (2014) The pharmacodynamics of L-arginine. Altern Therap Health Med 20, 48-54.

59. Utagawa T (2004) Arginine metabolism: enzymology, nutrition, and clinical significance. J Nutr 134, 2854S-2857S.

60. Morris SM Jr (2007) Arginine metabolism: boundaries of our knowledge. J Nutr 137, 1602S-1609S. 